\title{
Triclosan Suppresses Testicular Steroidogenesis via the miR-6321/JNK/ Nur77 Cascade
}

\author{
Mei Ha ${ }^{a}$ Pei Zhang ${ }^{b} \quad$ Lianbing Lic Changjiang Liuc \\ aSchool of Nursing, Chongqing Medical and Pharmaceutical College, Chongqing, bepartment of \\ Gastrointestinal Surgery, Union Hospital, Tongji Medical College, Huazhong University of Science and \\ Technology, Wuhan, 'Key Lab of Birth Defects and Reproductive Health of National Health and Family \\ Planning Commission, Chongqing Population and Family Planning Science and Technology Research \\ Institute, Chongqing, China
}

\section{Key Words}

miR-6321 • Nur77 • JNK/C-Jun pathway • Testicular steroidogenesis • Triclosan

\begin{abstract}
Background/Aims: Triclosan (TCS), a broad-spectrum antibacterial and antifungal compound and an endocrine disruptor, has anti-androgenic properties and could adversely affect male reproduction and fertility. Methods: To elucidate the underlying roles of miRNAs and the MAPK pathway in TCS-mediated repression of testicular steroidogenesis, Sprague-Dawley male rats were dosed daily with TCS for 31 days, and TM3 cells were exposed to TCS for $24 \mathrm{~h}$ after the pretreatments with the activator of JNK, Nur77 siRNA, or recombinant lentivirus vector for Nur77. Tissues and/or cells were analyzed by several techniques including transmission electron microscopy, lentivirus production, overexpression, gene silencing, luciferase reporter assay, chromatin immunoprecipitation, western blot, and real-time PCR. Results: TCS caused histopathologic alterations in the testis and reduced plasma LH and testicular testosterone. TCS induced miR-6321 expression, which in turn depressed its target gene, Map3k1. The inhibition of Map3k1 subsequently inactivated its downstream JNK/c-Jun pathway. ChIP and GPCR assays confirmed that c-Jun directly bound to the Nur77 DNA promoter regions to regulate Nur77 expression. The knockdown and overexpression of Nur77 demonstrated that the JNK/C-Jun-mediated decline in the transcription and translation of Nur77 resulted in the depression of steroidogenic proteins including SRB1, StAR, and 3 $\beta-H S D$. Intriguingly, the protein expressions of $5 \alpha$-Reductases (SRD5A1 and SRD5A2) were also downregulated after TCS exposure. Conclusion: Taken together, the miR-6321/Map3k1-regulated JNK/c-Jun/ Nur77 cascade contributes to TCS-caused suppression of testicular steroidogenesis, and the decrease in $5 \alpha$-Reductase expressions may be the compensatory mechanism.
\end{abstract}

M. Ha and P. Zhang contributed equally to this work.

\begin{tabular}{ll}
\hline Dr. Changjiang Liu & Chongqing Population and Family Planning Science and Technology Research Institute \\
& 18 Honghuang Road, Chongqing 400020 (China) \\
& Tel. +862386715335, Fax +862386715335, E-Mail cj_514@163.com
\end{tabular}




\section{Cellular Physiology Cell Physiol Biochem 2018;50:2029-2045 and Biochemistry \begin{tabular}{l|l} 
DOI: 10.1159/000495049 & (c) 2018 The Author(s). Published by S. Karger AG, Basel \\
www.karger.com/cpb
\end{tabular}

\section{Introduction}

Triclosan (TCS) is a broad-spectrum antibacterial and antifungal compound that has been extensively used in various personal care and industrial products, such as cosmetics, textile goods, and medical devices. Due to its widespread use and bioaccumulation, TCS has been detected in human blood, urine, breast milk, and amniotic fluid [1, 2]. Analyses of the chemical structure indicate that TCS has similar structures to bisphenol A, dioxins, polychlorinated biphenyls, etc., suggesting that they may have similar chemical properties [3]. Human exposures and the structural similarity have raised concerns about the detrimental effects of TCS, such as developmental toxicity [4], thyrotoxicity [5], neurotoxicity [6], and gut microbiome perturbation [7]. Moreover, accumulating evidence has shown that TCS has anti-androgenic properties and could induce reproductive toxicity $[8,9]$.

The reproductive toxicity of TCS has been studied for many years and investigations in humans and animals have demonstrated that TCS could adversely affect mammalian reproduction and fertility, testicular physiology and function, and steroid hormone production, etc [10]. Kumar et al. [11] reported that TCS exposure led to extensive histopathological malformations in rat testes and significant decreases in LH, FSH, and testosterone. Testicular steroidogenesis in Leydig cells plays an essential role in male reproduction and fertility, and some possible mechanisms by which TCS inhibits testicular steroidogenesis have been proposed in recent years. The study suggested that the main factor responsible for testosterone decline was TCS-induced cAMP deprivation, which was caused by the disruption of adenylyl cyclase enzyme activity [12]. In their further study, Kumar et al. [11] thought that the transcriptional repression of testicular steroidogenic enzymes (StAR, P450scc, P450c17, 3 $\beta$-HSD, and 17 $\beta$-HSD) also contributed to the downregulation of testosterone. Another study in TCS-exposed pregnant rats reported that the increase in mRNA levels of steroid metabolism enzymes, including Ugt1a1, Sult1e1, and $5 \alpha$-Reductases accelerated the metabolism of steroid hormones, leading to the decrease in testosterone and progesterone [13]. Moreover, displacing testosterone from its natural hormone receptor by competitively binding to the receptor was considered as another potential mechanism by which TCS disturbed the hypothalamic-pituitary-testis axis (HPT axis) and eventually inhibited testosterone biosynthesis [10].

Testicular steroid hormone synthesis and secretion are modulated by the HPT axis and multiple signaling pathways are involved in the molecular events. Mitogen-activated protein kinases (MAPKs), characterized as proline-directed serine-threonine kinases, include three major subfamilies: ERK, JNK, and p38. MAPKs are important cellular signaling components which transmit various extracellular stimuli into intracellular response through serial phosphorylation cascades. Accumulating literatures have indicated that the MAPK pathway is closely involved in the regulation of steroidogenesis in response to various extracellular factors. In primary rat Leydig cells, orexin A induced $3 \beta$-HSD expression and stimulated testosterone production via the ERK and p38 pathways [14]. The study by Lai et al. [15]. reported that fibroblast growth factor 9 could specifically activate the ERK pathway in normal mouse Leydig cells and the JNK and ERK pathways in MA-10 mouse Leydig tumor cells to stimulate steroidogenesis. Our previous study also found that the ERK pathway played a critical role in DEHP-caused suppression of testosterone production. In detail, ERK induced $5 \alpha$-Reductase 2 that catalyzed the irreversible conversion of testosterone to $5 \alpha$-DHT in the male reproductive tract [16]. Additionally, in recent years prominent roles of miRNAs have been uncovered in regulating steroidogenesis in Leydig cells. The study by $\mathrm{Hu}$ et al. [17] reported that miR-125a and miR-455 negatively regulated SRB1-mediated selective delivery of HDL cholesterol in Leydig cells and consequently inhibited HDL-supported steroidogenesis. Another study observed that StAR expression and steroidogenesis were negatively modulated by miR-150 in primary mouse Leydig cells [18]. However, whether the MAPK pathway and/or miRNAs function significantly in TCS-caused repression of testicular steroidogenesis is still unknown. Therefore, in order to reveal the underlying roles of the MAPK pathway and miRNAs, Sprague-Dawley male rats and TM3 cells (mouse Leydig cell 


\section{Cellular Physiology Cell Physiol Biochem 2018;50:2029-2045

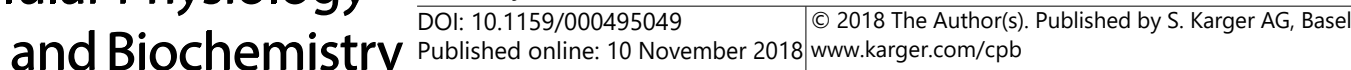

Ha et al.: The miR-6321/JNK/Nur77 Inhibits Steroidogenesis

line) were used in this study. The findings demonstrate that the miR-6321/Map3k1-mediated JNK/c-Jun/Nur77 cascade contributes to the declines in testicular steroid hormones after TCS exposure, and our study will be helpful for understanding the mechanisms by which TCS interferes with testicular steroidogenesis.

\section{Materials and Methods}

\section{Animals and treatments}

Twenty-four male Sprague-Dawley rats (23 days old) were purchased from the Daping Hospital Animal Laboratory (Chongqing, China). Rats were randomly divided into four groups ( $\mathrm{n}=6 /$ dose group) by counterbalancing body weights before treatments and were housed in an ambient temperature $(22 \pm$ $1{ }^{\circ} \mathrm{C}$ ), relative humidity ( $50 \pm 5 \%$ ), and light (12-h light/dark cycle) controlled room. Feed and water were provided ad libitum. Animals were gavaged daily for 31 days with TCS (50, 100, and $200 \mathrm{mg} / \mathrm{kg} /$ day; DR Co., Germany) or corn oil (vehicle control). The dosing volume was readjusted daily according to body weights of rats. Treatments were carried out between 0800 and $1000 \mathrm{~h}$. Experimental protocols used including the rat age, and the exposure time and route were based on recommendations of the US EPA Endocrine Disrupter Screening and Testing Advisory Committee; the exposure dose was based on the oral LD50 (5000 mg/kg) in rats. Animal experiments were ratified by the Research Ethics Committee of Chongqing Population and Family Planning Science and Technology Research Institute.

\section{Histopathologic evaluation}

Histopathologic alterations in rat testes were assessed by hematoxylin and eosin (HE) staining analysis and transmission electron microscope (TEM) analysis. For HE analysis, testes were fixed in $4 \%$ paraformaldehyde for $24 \mathrm{~h}$. Then fixed testes were embedded, sliced, and stained with HE. For TEM analysis, testes were fixed in $2.5 \%$ glutaraldehyde at $4{ }^{\circ} \mathrm{C}$ for $24 \mathrm{~h}$ and diced. Slices of testes were washed in icecold cacodylate buffer and then postfixed in $1 \%$ osmium tetroxide in phosphate buffer. Then ultrastructural changes in testes were evaluated by a FEI Tecnai 12G2 transmission electron microscope.

\section{Hormone determination}

GnRH, LH, FSH, and testosterone levels as well as cholesterol levels in rat plasma were determined using enzyme-linked immunoabsorbent assay kits (Assay Designs Inc., USA). Neither significant crossreactivity nor interference was noted in detections. All samples were assayed in duplicate.

\section{Cell culture}

TM3 cells (mouse Leydig cell line) were purchased from the Chinese Academy of Science and grown in DMEM medium supplemented with $10 \%$ fetal bovine serum in a humidified incubator $\left(37^{\circ} \mathrm{C}, 5 \% \mathrm{CO}_{2}\right)[16]$. Anisomycin (Anis.; Abcam Inc., USA), a potent activator of JNK, was prepared in DMSO. After pretreatments with $100 \mu \mathrm{M}$ of Anis for $1 \mathrm{~h}$, TM3 cells were incubated with $10 \mu \mathrm{M}$ of TCS for $24 \mathrm{~h}$ [5].

\section{Cell viability assay}

After treatments with diverse concentrations of TCS in 96-well plates for $24 \mathrm{~h}$, TM3 cells were incubated with $10 \mu \mathrm{l}$ of Cell Counting Kit-8 solution (Dojindo Laboratories, Japan) at $37^{\circ} \mathrm{C}$ for $1 \mathrm{~h}$. The absorbance was detected at $450 \mathrm{~nm}$.

\section{Luciferase reporter assay}

TargetScan software was used to identify putative miR-142-5p and miR-6321 binding sites in Map3k1. The wide-type and mutant Map3k1 3'-UTR fragments were cloned into the pmirGLO reporter vector (Promega, USA) to generate recombinant vectors including pmirGLO-Map3k1-wt and pmirGLO-Map3k1$\mathrm{mt}$. Sequences of the recombinant plasmids were confirmed before transfections. For the luciferase reporter assay, TM3 cells were transiently co-transfected with miRNAs (miR-142-5p mimic, inhibitor, or scrambledmiR-142-5p negative control; miR-6321 mimic, inhibitor, or scrambled-miR-6321 negative control) and reporter vectors (wild-type reporter vector or mutant-type reporter vector) using Lipofectamine 2000 


\section{Cellular Physiology Cell Physiol Biochem 2018;50:2029-2045 and Biochemistry Published \begin{tabular}{l|l} 
DOI: 10.1159/000495049 2018 The Author(s). Published by S. Karger AG, Basel \\
was.karger.com/cpb
\end{tabular}}

Ha et al.: The miR-6321/JNK/Nur77 Inhibits Steroidogenesis

(Life Technologies Corp., USA). The relative luciferase activity was determined using the dual-luciferase reporter assay system (Promega, USA) according to the manufacturer's protocols at $24 \mathrm{~h}$ post-transfection. All experiments were performed in triplicate.

\section{Chromatin immunoprecipitation (ChIP) assay}

ChIP assays were carried out using ChIP kits (Millipore, USA). TM3 cells were incubated with 1\% formaldehyde for $10 \mathrm{~min}$ at $37^{\circ} \mathrm{C}$ to cross-link DNA and associated proteins, and then were sonicated to make soluble chromatin with DNA fragments. The chromatin fragments were immunoprecipitated with anti-c-Jun (Cell Signal Technology, USA), anti-RNA Polymerase II (the positive control; Millipore, USA), or normal rabbit IgG (the negative control; Cell Signal Technology, USA) for $12 \mathrm{~h}$ at $4{ }^{\circ} \mathrm{C}$. The chromatin-antibody-protein A agarose complexes were subsequently washed with low salt, high salt, LiCl, and Tris/EDTA buffers, and protein-DNA complexes were eluted. $\mathrm{NaCl}$ was added to the eluates that were then incubated for $4 \mathrm{~h}$ at $65^{\circ} \mathrm{C}$ to reverse the formaldehyde cross-linking. After treatments with protease $\mathrm{K}$ for $1 \mathrm{~h}$ at $45^{\circ} \mathrm{C}$, samples were extracted with phenol-chloroform and precipitated with ethanol. The recovered DNA was resuspended in TE buffer and used for qPCR amplification. The proximal Nur77 promoter primers were as follows: forward: 5'-CTTGCAGGAGCTGGGAAGTC-3' and reverse: 5'-GGGACAGATCCAACGGAATCAC-3'. PCR products (224 bp) were analyzed on $2 \%$ agarose gels.

\section{Nur77 gene silencing}

TM3 cells grown to 70\% confluent were transfected with the Nur77 siRNA (Santa Cruz Biotechnology Inc., USA) or the scrambled siRNA (the negative control; Life Technologies Corp., USA). The siRNAs prepared in nuclease-free sterile water were diluted to $400 \mathrm{nM}$ with Opti-MEM Medium. The siRNAs were transfected using Lipofectamine RNAiMAX reagents (Life Technologies Corp., USA) following manufacturer's instructions. Transfected cells were incubated at $37{ }^{\circ} \mathrm{C}$ for $24 \mathrm{~h}$ and then the transfection efficiency was evaluated using western blot. Nur77 antibody was from the Abcam Inc. (USA).

\section{Lentivirus production and infection}

The cloning vector (pCDH-Puro), E.Z.N.A. Gel Extraction Kit, Plasmid DNA Mini Kit I and Endo-free Plasmid DNA Mini Kit II (Omega Bio-tek, Inc., USA), and Lipofectamine 2000 transfection reagent (Life Technologies Corp., USA) were used to establish the Leydig cell line with stable Nur77 overexpression. pCDH-Puro-Nur77 (the recombinant lentivirus vector) and pCDH-Puro (the blank vector as the negative control) were packaged into mature lentivirus by 293T cells and used to infect TM3 cells. The efficiency of Nur77 overexpression was determined using real-time PCR and western blot. Primer sequences of Nur77 were as follows: forward: 5'-GCGAGTCCGAGGATGGTG-3' and reverse: 5'-CGGCCTCGCTCTGATTGTAG-3'. Nur77 antibody was from the Abcam Inc. (USA).

RNA or miRNA isolation, and quantitative real-time PCR

Total RNA was extracted from rat testes and TM3 cells using Trizol reagents (Invitrogen, USA). RNA was converted to cDNA using SuperScript VILO cDNA synthesis kits (Life Technologies Corp., USA). RT-PCR was performed using Platinum SYBR Green qPCR SuperMix-UDG with ROX (Invitrogen, USA). $\beta$-Actin was used as an internal control. Thermal cycling conditions comprised two initial steps at 50 ${ }^{\circ} \mathrm{C}$ for $2 \mathrm{~min}$ and $95{ }^{\circ} \mathrm{C}$ for $2 \mathrm{~min}$, followed by 40 cycles $\left(95{ }^{\circ} \mathrm{C}\right.$ for $15 \mathrm{sec}$ and $60{ }^{\circ} \mathrm{C}$ for $1 \mathrm{~min}$ ). Primer sequences of target genes were as follows: Map3k1 forward: $5^{\prime}$-ACCACCACTGCATGTCAATCTG-3 ${ }^{\prime}$ and reverse: 5'-CCTGATTCCTCCGCTGTGATC-3'; LHR forward: 5'-CTGCGCTGTCCTGGCC-3' and reverse: 5'-CGACCTCATTAAGTCCCCTGAA-3'. MicroRNA was extracted from rat testes using miRcute miRNA isolation kits (Qiagen, China). cDNA was synthesized using miRcute miRNA First-Strand cDNA synthesis kits (Qiagen, China). RT-PCR was performed using miRcute plus miRNA qPCR detection kits (Qiagen, China). U6 was used as an internal control. Thermal cycling conditions were as follows: initial denaturation at $95{ }^{\circ} \mathrm{C}$ for $15 \mathrm{~min}$, followed by 40 cycles $\left(94{ }^{\circ} \mathrm{C}\right.$ for $20 \mathrm{sec}$ and $60{ }^{\circ} \mathrm{C}$ for $34 \mathrm{sec}$ ). Primer sequences of target miRNAs were as follows: miR-142-5p: 5'-CCCGGCATAAAGTAGAAAGCACTA-3' and miR-6321: 5'-CCGTACTGCAGTGAGTTCTATGAA-3'. Samples were run in triplicate. 


\section{Cellular Physiology Cell Physiol Biochem 2018;50:2029-2045

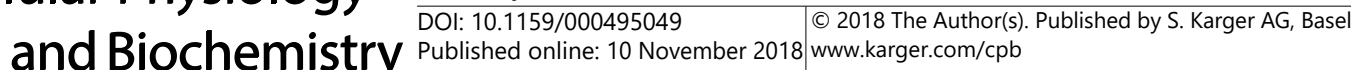 \\ Ha et al.: The miR-6321/JNK/Nur77 Inhibits Steroidogenesis}

Western blot analysis

Proteins were extracted from rat testes and TM3 cells using Cell Lysis Buffer for Western blot and IP. Concentrations of protein were determined using BCA protein assay kits (Beyotime Biotech Inc., China). Twenty $\mu \mathrm{g}$ of protein were separated and electrophoresed by SDS-PAGE. Nitrocellulose membranes were blocked by TBST containing $5 \%$ non-fat milk for $1 \mathrm{~h}$ at $37^{\circ} \mathrm{C}$, followed by incubations with specific primary antibodies overnight at $4{ }^{\circ} \mathrm{C}$ and HRP-conjugated secondary antibodies for $1 \mathrm{~h}$ at $37^{\circ} \mathrm{C}$. Target proteins were visualized and analyzed using the ECL system. ERK, p-ERK, JNK, p-JNK, p38, p-p38, P450scc, GAPDH, and $\beta$-Tubulin antibodies were bought from the Cell Signaling Technology Inc. (USA). Map3k1, SRD5A2, Nur77, c-Jun, p-c-Jun, SRB1, StAR, P450c17, and $\beta$-Actin antibodies were gotten from the Abcam Inc. (USA). AR, $\mathrm{ER} \alpha, \mathrm{SF}-1,3 \beta$-HSD, and $17 \beta$-HSD antibodies were purchased from the Santa Cruz Biotechnology Inc. (USA). SRD5A1, c-Fos, and p-c-Fos antibodies were obtained from the Bioworld Technology Inc. (China).

\section{Immunohistochemical (IHC) and immunofluorescent (IF) staining analysis}

Testes were fixed in $4 \%$ paraformaldehyde for $24 \mathrm{~h}$. After the microwave antigen retrieval, sections were deparaffinized, rehydrated, and blocked in 3\% goat serum. For IHC analysis, sections were incubated with anti-p-JNK (Cell Signaling Technology Inc., USA) overnight at $4{ }^{\circ} \mathrm{C}$, followed by treatments with biotinylated anti-rabbit IgG, peroxidase-conjugated streptavidin, diaminobenzidine substrate, and hematoxylin. For IF analysis, sections were incubated with anti-Nur77 (Abcam Inc., USA) overnight at $4{ }^{\circ} \mathrm{C}$, followed by incubations with FITC-coupled goat anti-rabbit IgG for $1 \mathrm{~h}$ in dark. Nuclei were stained with DAPI for 5 min. Likewise, SRB1 protein expression in cells was also detected using IF analysis.

\section{Statistical analysis}

Quantitative data were reported as mean \pm SD. Significance was analyzed by one-way analysis of variance (ANOVA) with least-significant difference (LSD) following appropriate transformations to the normal distribution and equal variance where necessary. The difference was considered significant when $P<0.05$.

\section{Results}

Triclosan reduced the relative testis and epididymis weight of exposed animals

Neither clinical signs of toxicity nor mortalities were noted during the period of TCS treatment. Animals had normal activities and food consumption. The body weight and weight gain were not significantly affected by TCS. However, TCS-treated rats exhibited signs of testicular atrophy, as indicated by a dose-dependent decrease in the relative testis $(P=$ $0.030)$ and epididymis weight $(P=0.046$; Fig. 1A).

\section{Triclosan declined steroid hormones}

A dose-dependent downregulation in plasma testosterone and LH levels was observed after TCS exposure. Compared with the control, testosterone level in the medium- and highdose group was reduced by $13.9 \%(P=0.013)$ and $23.5 \%(P=0.002)$, respectively. Moreover, $\mathrm{LH}$ level in TCS-treated rats was lower than that of the control $(P<0.001)$. However, GnRH, FSH, and cholesterol levels were not significantly affected by TCS (Fig. 1B).

\section{Triclosan led to histopathologic changes in rat testes}

HE staining analysis uncovered apparent histological alterations in the testis after TCS exposure. Normal spermatogenesis and seminiferous tubules filled with spermatogonia, spermatocytes, and spermatids were observed in the control and low-dose TCS group. After treatments with 100 and $200 \mathrm{mg} / \mathrm{kg}$ of TCS, however, partial seminiferous tubules were shrunk, accompanied by narrowed diameters, collapsed structures, and disordered cell arrangements (Fig. 1C). Ultrastructural changes caused by TCS were further evaluated by TEM analysis. Normal organelles including nucleus, nucleoli, chromatin, endoplasmic reticulum, and mitochondria were observed in the control. However, TCS treatment resulted in significant ultrastructural alterations, as indicated by deformed nucleus, aggregated 


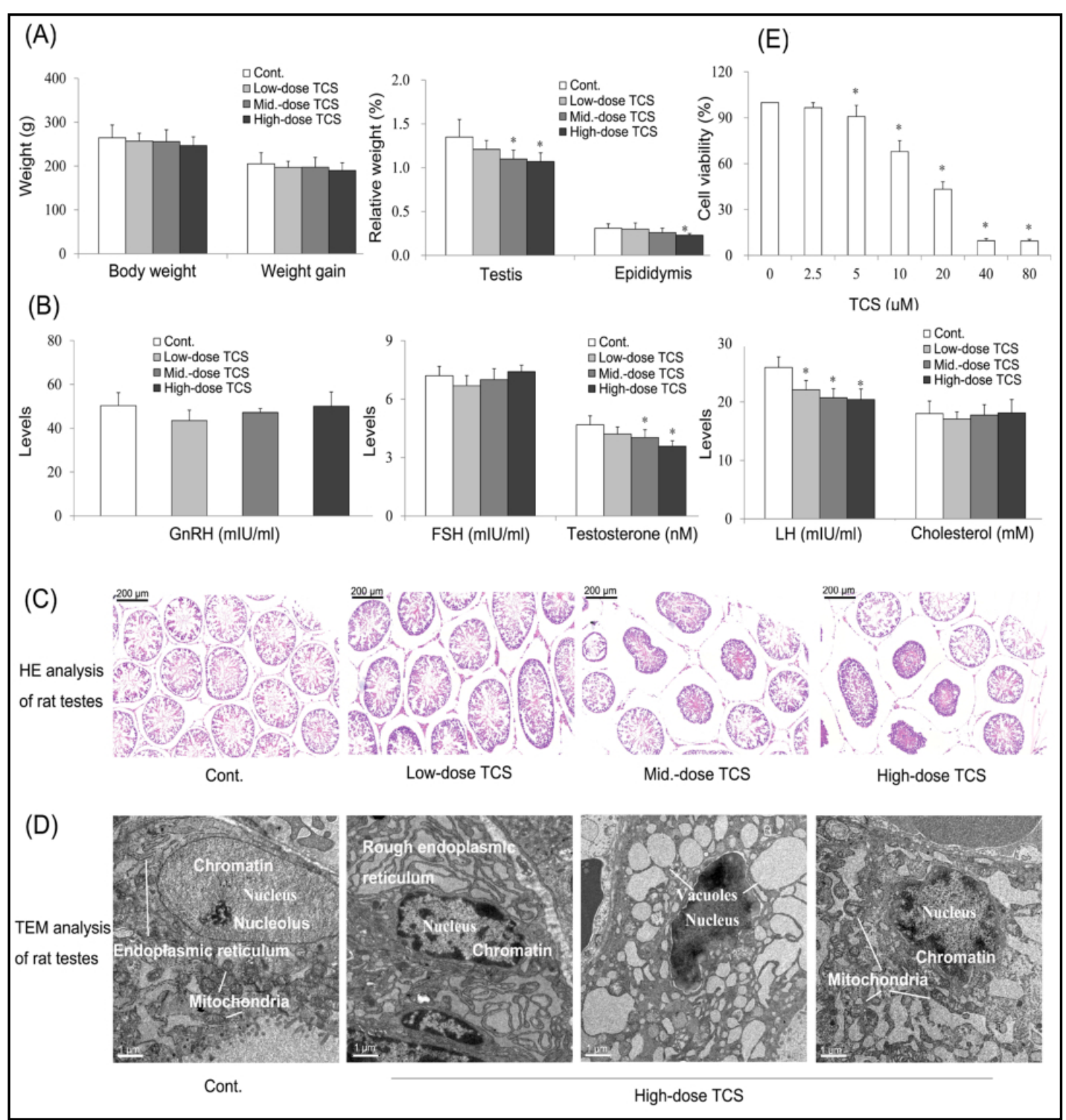

Fig. 1. Effects of TCS on the testicular steroid hormones and histopathologic changes in rat testes. (A) Effects of TCS on body, testis, and epididymis weight. TCS decreased the relative weight of the testis and epididymis. The relative weight was \% body weight. (B) Effects of TCS on levels of GnRH, FSH, testosterone, LH, and cholesterol. TCS declined LH and testosterone levels in rat plasma. (C) HE analysis of rat testes. Histological alterations in the testis were evaluated by HE staining. Magnification: $\times 100$. (D) TEM analysis of rat testes. Ultrastructural alterations in the testis were assessed by TEM analysis. Magnification: $\times 10000$. (E) TCS suppressed the viability of TM3 cells. ${ }^{*} \mathrm{P}<0.05$, compared with the control.

chromatin, multiple vacuoles, swollen mitochondria and vague cristae, as well as dilated rough endoplasmic reticulum (Fig. 1D).

Triclosan restrained the viability of Leydig cells

Cytotoxic effects of TCS on Leydig cells were observed and treatments with diverse concentrations of TCS led to a significant loss of cell viability. After exposure to 5 and $10 \mu \mathrm{M}$ of TCS for $24 \mathrm{~h}$, cell viability was reduced to $90.85 \%(P=0.001)$ and $69.91 \%(P<0.001)$, respectively (Fig. 1E). 


\section{Cellular Physiology Cell Physiol Biochem 2018;50:2029-2045

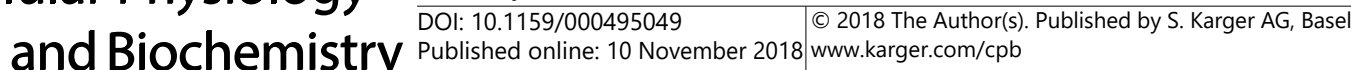 \\ Ha et al.: The miR-6321/JNK/Nur77 Inhibits Steroidogenesis}

Triclosan induced miR-142-5p and miR-6321 expressions in rat testes

After a preliminary screening for differentially expressed miRNAs in testes by miRNA microarray analysis (data not shown), two significantly upregulated miRNAs were screened out and further confirmed using quantitative real-time PCR. TCS induced miR-142-5p and miR-6321 expressions, with the maximum inductive effect observed in the high-dose group. A 2.4-fold increase in miR-142-5p $(P=0.012)$ and a 2.0-fold increase in miR-6321 $(P=0.018)$ in the high-dose group were observed compared with the control (Fig. 2A).

miR-6321 directly targeted Map3k1 3'-UTR in TM3 cells

The bioinformatics prediction using TargetScan showed that Map3k1 was the direct target of miR-142-5p and miR-6321 (Fig. 2B). To confirm whether miR-142-5p and miR6321 could directly target the 3 '-UTR of Map3k1, luciferase reporter assays were performed in TM3 cells. The gain-of-function and loss-of-function studies displayed that the luciferase activity of the reporter gene harboring 3'-UTR of Map3k1 was suppressed by miR-6321 mimic $(P<0.001)$ and induced by miR-6321 inhibitor $(P=0.027)$. However, miR-6321 mimic and inhibitor had no significant effects on the luciferase activity of the reporter gene containing mutant 3'-UTR of Map3k1. Moreover, miR-142-5p mimic and inhibitor did not significantly affect the luciferase activity of the reporter gene containing wild-type or mutant 3'-UTR of Map3k1 (Fig. 2C).

\section{Triclosan inhibited the Map3k1/JNK/c-Jun pathway}

TCS treatment inhibited mRNA and protein expressions of Map3k1 in the testis. Map3k1 mRNA level displayed a dose-dependent decreasing trend $(P=0.004)$. Compared with the control, Map3k1 protein expression in the medium- and high-dose group was attenuated by $38.9 \%(P=0.042)$ and $46.9 \%(P=0.016)$, respectively (Fig. 2D). Map3k1, a MAPKKK, functions as an upstream activator in the JNK pathway and is required for JNK activation. The present study found that Map3k1 suppression led to the inactivation of the JNK pathway, resulting in the inhibition of JNK phosphorylation, especially in the high-dose group $(P=$ 0.003); nevertheless, no significant changes in protein expressions of ERK, p-ERK, p38, and p-p38 were observed after TCS exposure (Fig. 2E). IHC analysis showed the similar decreasing trend of p-JNK in rat testes (Fig. 2F). The inactivated JNK pathway subsequently suppressed its downstream activator protein 1 (AP-1). c-Jun, the substrate of JNK and also a critical member of the AP-1 family, was downregulated following the JNK inactivation, as indicated by the decreased $p$-c-Jun protein expression $(P<0.001)$. However, another member of the AP-1 family, c-Fos, was not significantly affected by the JNK pathway (Fig. 3A).

TCS also inhibited the Map3k1/JNK/c-Jun pathway in TM3 cells. After treatments with $10 \mu \mathrm{M}$ of TCS for $24 \mathrm{~h}$ in TM3 cells, Map3k1 protein level was downregulated by $32.7 \%$ compared with the control $(P=0.004)$. Moreover, protein levels of $\mathrm{p}$-JNK and p-c-Jun also displayed a concentration-dependent decrease $(P=0.036$ and 0.021 , respectively). Activated signs of the ERK and p38 pathways were not observed in vitro after TCS exposure (Fig. 3B, C).

\section{Triclosan repressed Nur77 protein expression via the JNK/c-Jun pathway}

Effects of TCS on orphan nuclear receptors Nur77 as well as steroidogenic factor 1 (SF1) were evaluated, results demonstrating that TCS repressed Nur77 protein expression, which was attenuated by $36.4 \%$ in the high-dose group relative to the control $(P=0.002)$. The similar downregulating trend of Nur77 expression was also observed in rat testes by IF analysis. However, TCS had little effects on SF-1 (Fig. 3D).

To elucidate roles of the JNK/c-Jun pathway on Nur77 expression, Anis (a potent activator of JNK) was used to activate JNK first. The phosphorylation of JNK and c-Jun was induced after treatment with $100 \mu \mathrm{M}$ of Anis. In TM3 cells co-treated with TCS plus Anis, protein levels of p-JNK and p-c-Jun were elevated by $191.6 \%(P<0.001)$ and $150.0 \%(P$ $=0.006)$ compared with TCS-treated cells, respectively. After activations of the JNK/c-Jun pathway, Nur77 protein expression was recovered by $44.8 \%$ in the co-treated group (TCS plus Anis) compared with the TCS-treated group $(P=0.035)$. However, c-Fos, $\mathrm{p}$-c-Fos, and 


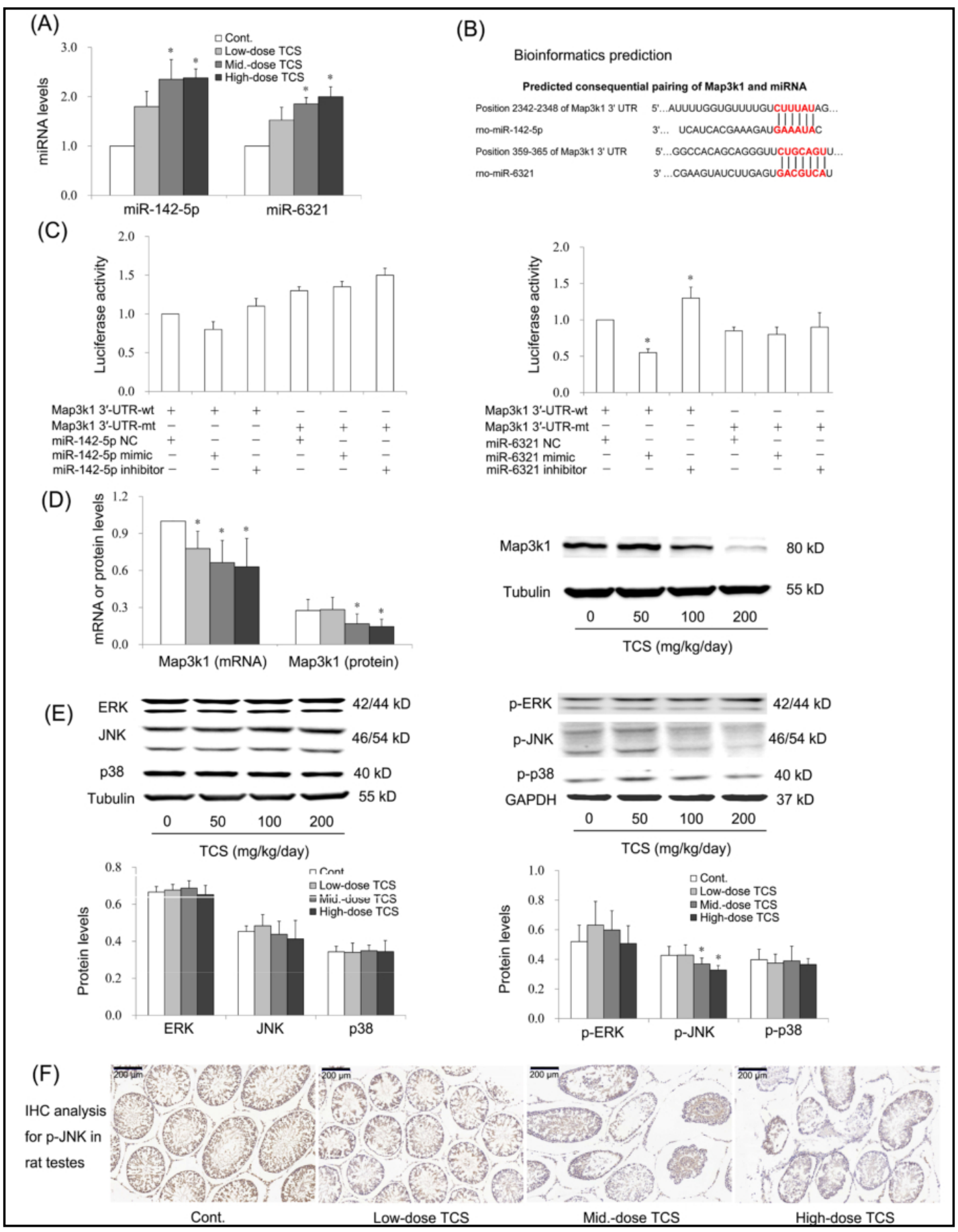

Fig. 2. Effects of TCS on miRNA expressions and the Map3k1/JNK pathway in rat testes. (A) Effects of TCS on miRNA expressions. TCS significantly induced miR-142-5p and miR-6321. (B) Bioinformatics prediction. TargetScan software was used to identify putative miR-142-5p and miR-6321 binding sites in Map3k1. (C) Luciferase reporter assay. The relative luciferase activity was determined using the dual-luciferase reporter assay system, results demonstrating that miR-6321 directly targeted Map3k1 3'-UTR in TM3 cells. (D) Effects of TCS on mRNA and protein expressions of Map3k1. TCS inhibited mRNA and protein expressions of Map3k1. (E) Effects of TCS on the MAPK pathway in the testis. TCS suppressed JNK phosphorylation, but had no significant effects on the ERK and p38 pathways. (F) IHC analysis for p-JNK. IHC analysis showed the similar decreasing trend of $\mathrm{p}$-JNK in rat testes. The brown indicated p-JNK positivity. Magnification: $\times 100$. ${ }^{*} \mathrm{P}<0.05$, compared with the control. 


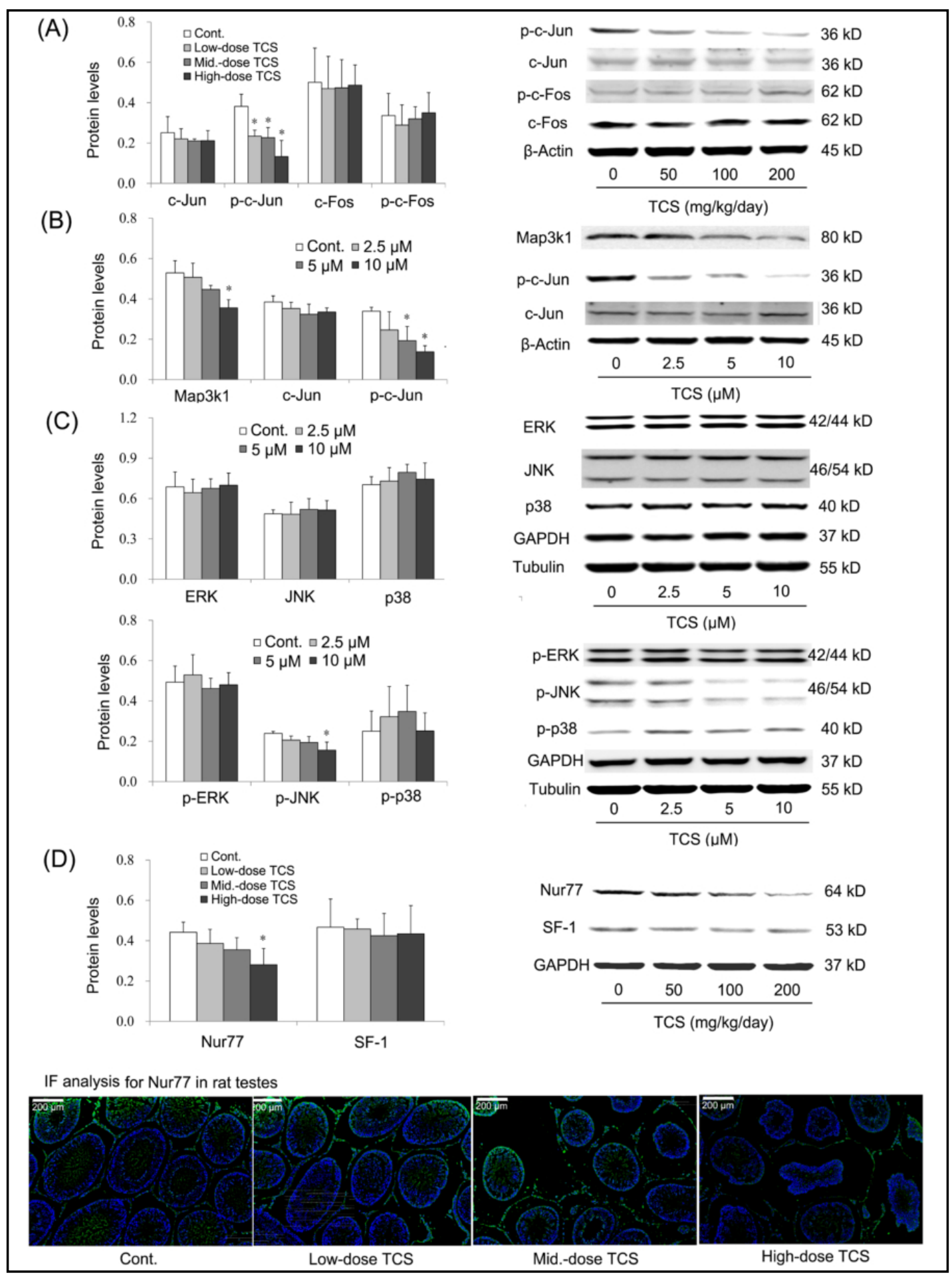

Fig. 3. Effects of TCS on the expressions of transcription factors in vivo and the MAPK pathway in vitro. (A) Effects of TCS on protein levels of c-Jun and c-Fos. TCS repressed c-Jun phosphorylation, having little effects on c-Fos in rat testes. (B) Effects of TCS on protein levels of Map3k1 and c-Jun. TCS downregulated protein expressions of Map3k1 and p-c-Jun in TM3 cells. (C) Effects of TCS on the MAPK pathway. TCS inactivated the JNK pathway in TM3 cells. (D) Effects of TCS on Nur77 and SF-1. TCS suppressed Nur77 protein expressions in the testis, and IF analysis of Nur77 exhibited the similar decreasing trend. The green indicated Nur77 positivity and nuclei were stained with DAPI (blue). Magnification: $\times 100 .{ }^{*} \mathrm{P}<0.05$, compared with the control. 
Fig. 4. Effects of TCS on the JNK cascade with / without Anis in vitro and on critical steroidogenic proteins in vivo. (A) Effects of TCS on the JNK pathway in the presence/ absence of Anis in TM3 cells. Treat me n t s with Anis (Anisomycin, a potent activator of JNK) significantly activated the J N K / c - J u n / Nur77 cascade. (B) ChIP assays of c-Jun and its binding motif. Anti-c-Jun, antiRNA polymerase II (positive control), and normal rabbit IgG (negative control) were used in the ChIP assays in TM3 cells. The binding activity was evaluated by qPCR. (C) Effects of TCS on steroidogenic proteins in the testis. Protein expres sion s of SRB1, StAR, 3ß-HSD, and

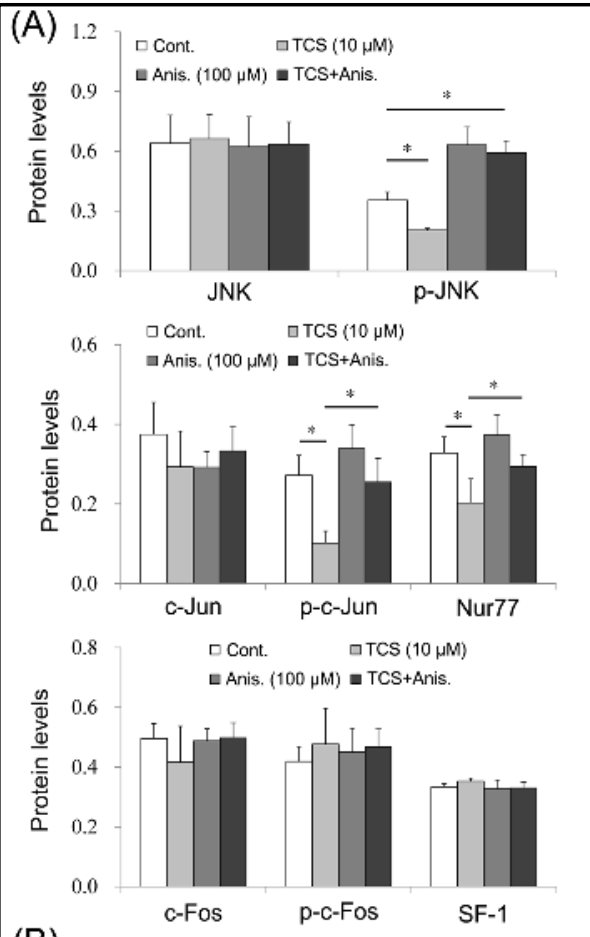

(B)

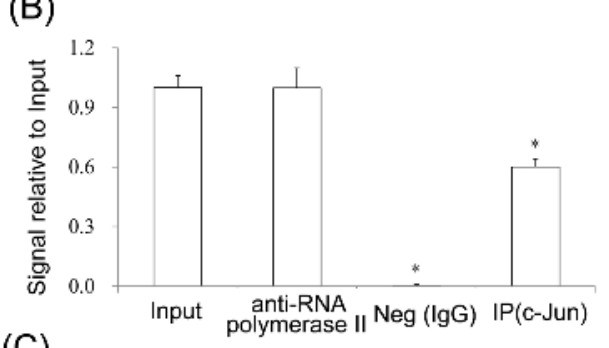

(C)
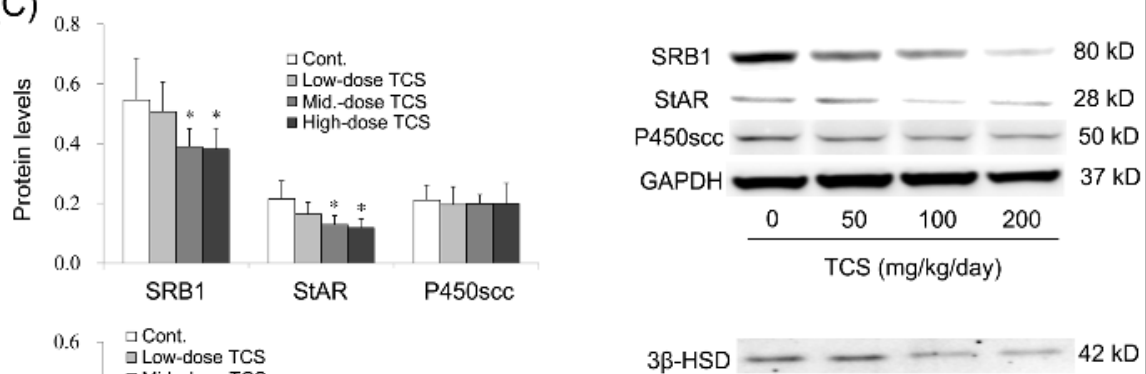

$17 \beta-\mathrm{HSD}=34.5 \mathrm{kD}$

$\mathrm{P} 450 \mathrm{c} 17-2-55 \mathrm{kD}$

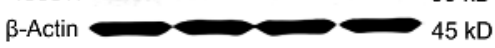

Tubulin

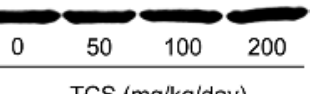

P450c17 were

inhibited after TCS exposure. ${ }^{*} \mathrm{P}<0.05$, compared with the control.

SF-1 protein expressions were not significantly affected by Anis (Fig. 4A). To further uncover the regulatory effect of c-Jun on Nur77 expression, ChIP and qPCR assays were subsequently performed in TM3 cells and results confirmed that c-Jun directly bound to the Nur77 DNA promoter regions to regulate Nur77 expression (Fig. 4B). 


\section{Cellular Physiology Cell Physiol Biochem 2018;50:2029-2045

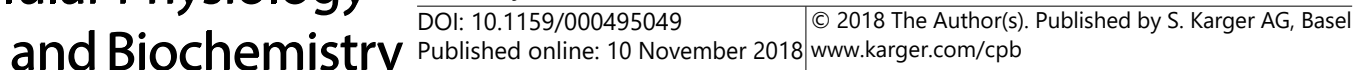 \\ Ha et al.: The miR-6321/JNK/Nur77 Inhibits Steroidogenesis}

\section{Triclosan downregulated steroidogenic protein expressions}

Four critical steroidogenic proteins, including SRB1, StAR, 3 $\beta$-HSD, and P450c17, were suppressed in rat testes after TCS exposure. Compared with the control, SRB1 protein expression in the medium- and high-dose group was declined by $28.6 \%(P=0.022)$ and $30.2 \%(P=0.016)$, respectively. Moreover, StAR, 3 $\beta$-HSD, and P450c17 protein levels in the high-dose group were reduced to $54.9 \%(P<0.001), 44.4 \%(P<0.001)$, and $52.3 \%(P=$ 0.001 ) of the control, respectively. However, no significant changes in P450scc and $17 \beta$-HSD were observed after TCS treatments (Fig. 4C).

\section{Nur77 regulated steroidogenic protein expressions}

To reveal the role of Nur77 on steroidogenic proteins, Nur77 gene silencing was performed first. After transfections with Nur77 siRNA, Nur77 protein expression was decreased by $65.2 \%(P<0.001)$, whereas the NC siRNA had little effects on Nur77 (Fig. $5 A)$. Upon knockdown of Nur77, protein expressions of SRB1, StAR, and 3 $\beta$-HSD were downregulated, with the maximum effect observed in the combined group (TCS plus Nur77 siRNA). After co-treatments with TCS plus Nur77 siRNA, SRB1, StAR, and 3 $\beta$-HSD protein expressions were suppressed by 52.0\% $(P=0.007), 82.8 \%(P<0.001)$, and $53.2 \%(P=$ 0.003), respectively. However, the inhibitory effect of TCS on P450c17 found in vivo was not observed in vitro. Likewise, Nur77 siRNA also failed to significantly affect P450scc and $17 \beta$-HSD in vitro (Fig. 5B).

To further verify the regulatory role of Nur77 on steroidogenic proteins, TM3 cells with stable Nur77 overexpression were established via the lentivirus transfection. Transfections of TM3 cells with the recombinant lentivirus vector for Nur77 augmented Nur77 expression at the mRNA and protein levels, and a 6.5-fold increase in the mRNA level $(P<0.001)$ and a 2.0 -fold increase in the protein level $(P=0.001)$ over the control were observed (Fig. $6 A)$. Upon overexpression of Nur77, protein expressions of SRB1, StAR, and 3 $\beta$-HSD were significantly upregulated. Compared with the TCS-treated regular TM3 cells, SRB1, StAR, and $3 \beta$-HSD protein expressions were elevated by $88.6 \%(P=0.001), 135.9 \%(P<0.001)$, and 92.2\% $(P<0.001)$, respectively, in TCS-treated Nur77-overexpressed TM3 cells. Nevertheless, overexpression of Nur77 had little effects on the protein expressions of P450c17, P450scc, and $17 \beta$-HSD (Fig. 6B).

\section{Triclosan suppressed LHR and AR expressions}

Expressions of LHR and AR both exhibited a dose-dependent reduction. Compared with the control, LHR mRNA level in the low-, medium-, and high-dose group was reduced to $71.2 \%(P=0.007), 61.3 \%(P=0.001)$, and $54.5 \%(P<0.001)$, respectively. Moreover, a $33.1 \%$ reduction in AR protein expression was noted in the high-dose group relative to the control $(P=0.015)$. Nevertheless, significant changes in ER $\alpha$ protein expression were absent from the current study (Fig. 6C).

\section{Triclosan lowered steroid $5 \alpha$-Reductase protein expressions}

TCS inhibited protein expressions of the steroid $5 \alpha$-Reductase (SRD5A1 and SRD5A2), with the maximum inhibitory effect observed in the high-dose group. SRD5A1 protein expression in the high-dose group was decreased by $45.3 \%$ in comparison with the control $(P=0.012)$ and SRD5A2 protein expression was decreased to $66.4 \%$ of the control $(P=$ 0.001 ; Fig. 6D). 


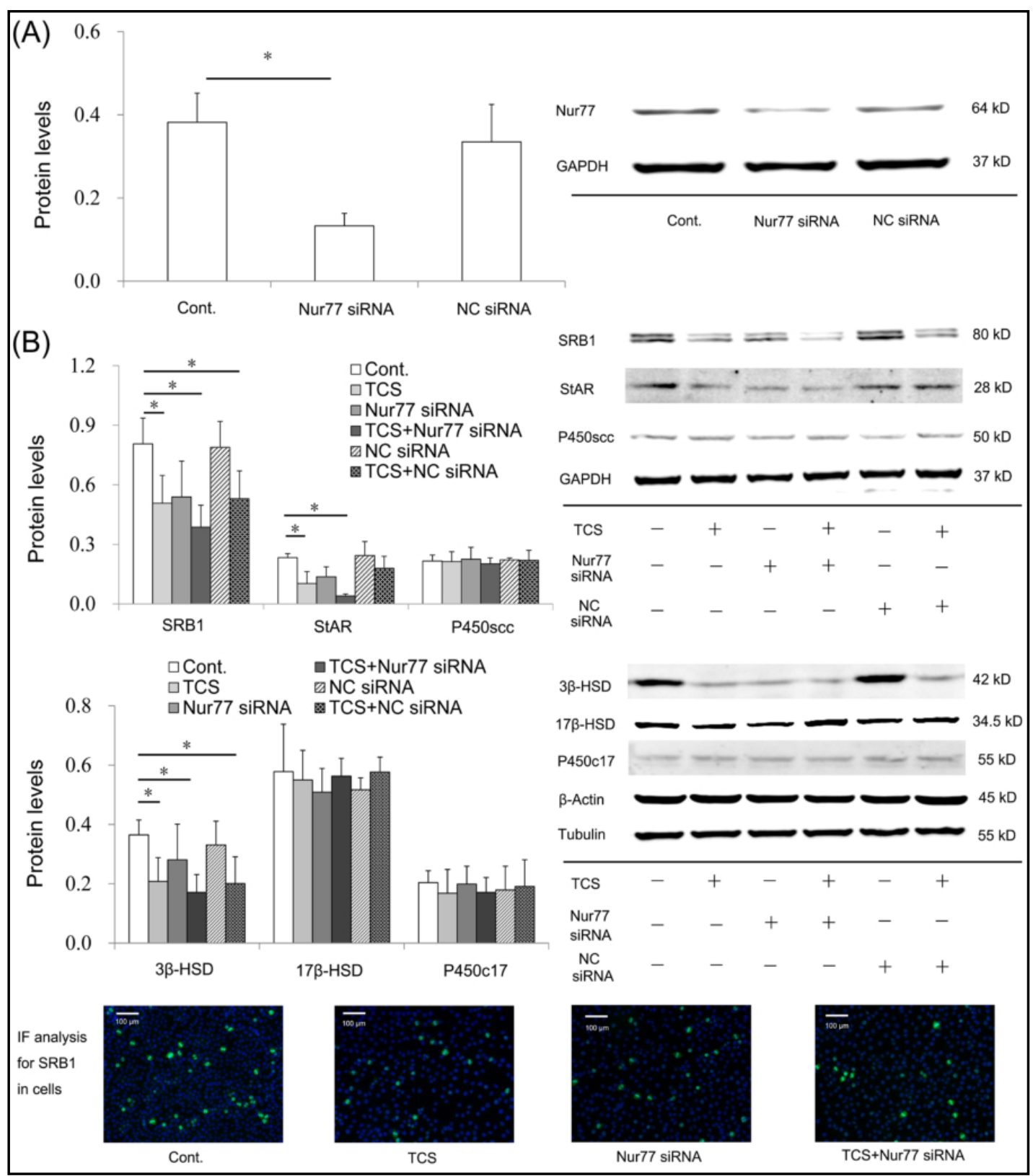

Fig. 5. Effects of TCS on the steroidogenic proteins with/without the specific Nur77 siRNA in TM3 cells. (A) Effects of Nur77 siRNA and NC siRNA on the protein level of Nur77. The specific Nur77 siRNA downregulated Nur77 protein expressions. (B) Effects of TCS on steroidogenic proteins with/without Nur77 siRNA in TM3 cells. Upon knockdown of Nur77, protein expressions of SRB1, StAR, and 3 $\beta$-HSD were decreased, but had no significant influence on the others. IF analysis for SRB1 expression in cells also displayed the downregulating trend. The green indicated SRB1 positivity and nuclei were stained with DAPI (blue). Magnification: $\times 200$. ${ }^{*} \mathrm{P}<0.05$, compared with the control.

\section{Discussion}

In this study, we explored the roles of miRNAs and the MAPK pathway in TCS-mediated repression of testicular steroidogenesis. The results indicate that TCS treatments lead to histopathologic alterations in rat testes and significant declines in plasma steroid hormones, and the miR-6321/Map3k1-modulated JNK/c-Jun/Nur77 cascade plays vital roles in this molecular event. 


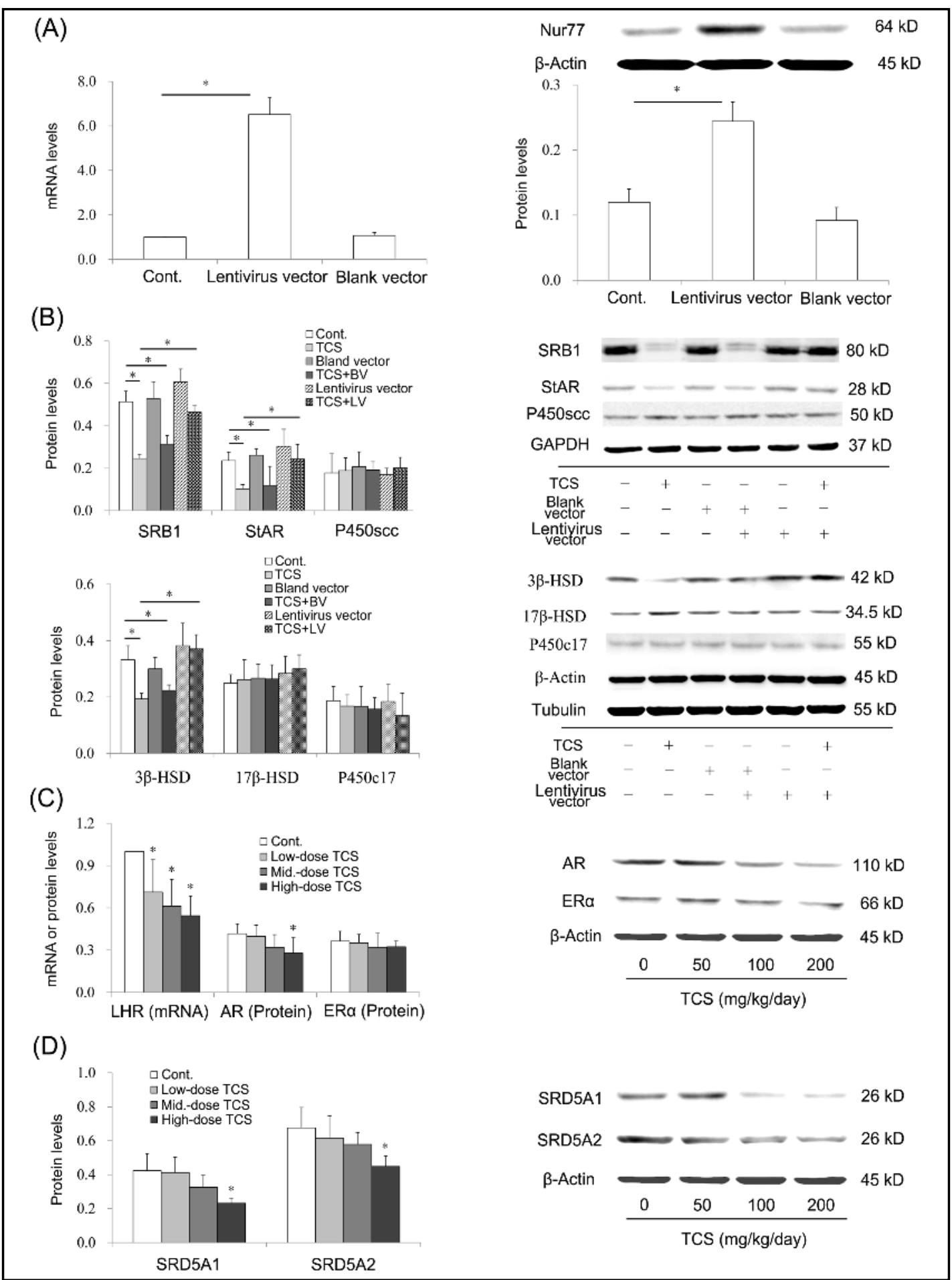

Fig. 6. Effects of TCS on the steroidogenic proteins with/without the recombinant lentivirus vector for Nur77 in TM3 cells. (A) Effects of the lentivirus vector for Nur77 on mRNA and protein levels of Nur77. The specific lentivirus vector for Nur77 upregulated mRNA and protein expressions of Nur77. (B) Effects of TCS on steroidogenic proteins with/without the lentivirus vector for Nur77 in TM3 cells. Upon overexpression of Nur77, protein expressions of SRB1, StAR, and 3 $\beta$-HSD were increased. (C) Effect of TCS on hormone receptors. TCS decreased LHR and AR level in rat testes. (D) Effect of TCS on $5 \alpha$-Reductases. Protein expressions of $5 \alpha$-Reductases (SRD5A1 and SRD5A2) were downregulated in the testis after TCS exposure. ${ }^{*} \mathrm{P}<0.05$, compared with the control. 


\section{Cellular Physiology Cell Physiol Biochem 2018;50:2029-2045

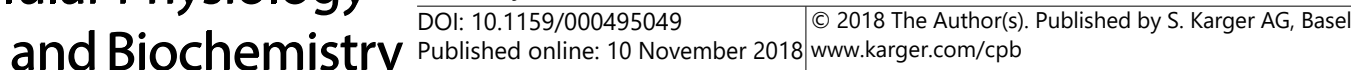 \\ Ha et al.: The miR-6321/JNK/Nur77 Inhibits Steroidogenesis}

In male reproduction and fertility, miRNAs play essential roles in processes of spermatogenesis such as spermatocyte meiosis, spermiogenesis, etc. by regulating expressions of target mRNAs $[19,20]$. However, studies on the roles of miRNAs in testicular Leydig cells are limited, especially after exposure to endocrine disruptors. After TCS treatments, two differentially high expressed miRNAs (miR-142-5p and miR-6321) in rat testes were screened out and further bioinformatics predictions and luciferase reporter assays confirmed that Map3k1 was the direct target of miR-6321. Map3k1 mRNA and protein expressions were downregulated, indicating that miR-6321 led to the mRNA degradation and translational inhibition of Map3k1 after TCS exposure. Map3k1 that functions as a MAPKKK and an upstream activator in the JNK pathway is required for JNK activation, and the repression of Map3k1 expression by TCS subsequently resulted in the inhibition of phosphorylation and inactivation of the JNK pathway. AP-1 is a transcription factor consisting of a group of immediate early genes, such as c-Jun and c-Fos, and it is also shown that AP-1 is the downstream substrate of the Map3k1/JNK pathway. In this study, targeted inhibition of the Map3k1/JNK pathway by TCS-induced miR-6321 resulted in decreased c-Jun phosphorylation; after the activation of the JNK pathway by Anis, c-Jun phosphorylation was subsequently induced. However, c-Fos, the other transcription factor of AP-1, was not influenced by the inactivated/activated JNK pathway after TCS treatment. This suggests that c-Jun is the true downstream effector of the JNK pathway in our current study. Hence, our study shows for the first time that the miR-6321-targeted inhibition of the Map3k1/JNK/cJun signaling pathway contributes to TCS-mediated suppression of testicular testosterone.

c-Jun has been demonstrated to modulate transcriptions of target genes by binding as homodimers or heterodimers to specific DNA sequences located in promoters of target genes, and the transcriptional activity of c-Jun is dependent on its degree of phosphorylation and abundance [21]. Inhibitions of c-Jun phosphorylation and declines in p-c-Jun protein expression impaired its transcriptional activity after TCS exposure, which in turn affected expressions of target genes, such as the orphan nuclear receptors. Orphan nuclear receptors including Nur77 and SF-1 are characterized as immediate early response genes expressed in various tissues including in hormonally stimulated steroidogenic cells. In our present study, when the JNK/c-Jun pathway was inactivated by miR-6321/Map3k1, Nur77 protein expression was downregulated; when the JNK/c-Jun pathway was activated by Anis, Nur77 protein expression was upregulated. ChIP and qPCR detections further validated that c-Jun could directly bind to the Nur77 DNA promoter regions to regulate Nur77 expression. However, SF-1 was not affected by the JNK/c-Jun pathway after TCS exposure. The study by Han et al. [22] also indicated that the JNK/c-Jun pathway positively modulated Nur77 nuclear export and activation. Therefore, our current study demonstrates that Nur77 is the direct downstream target gene of the Map3k1/JNK/c-Jun pathway and may be involved in this molecular event.

The orphan nuclear receptor Nur77, also as a transcription factor, has been shown to play essential roles in the transcription of steroidogenic enzymes-encoding genes and testosterone biosynthesis in Leydig cells [23]. It has been known that testicular steroidogenesis in Leydig cells includes conversions of cholesterol through several intermediate steroids into testosterone, and the processes are modulated and catalyzed by SRB1, StAR, P450scc, $3 \beta$-HSD, P450c17, and 17 $\beta$-HSD [16]. In this study, mRNA and protein expressions of SRB1, StAR, and 3 $\beta$-HSD were suppressed after TCS exposure, and then Nur77 knockdown and overexpression were performed in Leydig cells to further elucidate the regulatory effects of Nur77 on steroidogenic enzymes. When Nur77 was knocked down by the specific siRNA, protein expressions of SRB1, StAR, and 3 $\beta$-HSD were downregulated; when Nur77 was overexpressed by the recombinant lentivirus vector, protein expressions of SRB1, StAR, and $3 \beta$-HSD were upregulated. These data clearly indicate that SRB1, StAR, and 3 $\beta$-HSD are positively regulated by Nur77. In Leydig cells from 12-month-old rats, ERK-Nur77 regulated the transcription and expression of StAR gene [24]; after transfection with Nur77 expression vector, the promoter activities of NurRE and its natural targets (P450c17, 3 $\beta$-HSD, and StAR) were enhanced in K28 Leydig cells [23]. Therefore, our current study further demonstrates 


\section{Cellular Physiology Cell Physiol Biochem 2018;50:2029-2045 and Biochemistry \begin{tabular}{l|l} 
DOI: 10.1159/000495049 & (c) 2018 The Author(s). Published by S. Karger AG, Basel \\
www.karger.com/cpb
\end{tabular}

that JNK/c-Jun-mediated Nur77 indeed functions significantly in TCS-mediated repression of testosterone production.

Moreover, our current study suggests that the disturbance of the HPT axis also contributes to TCS-mediated repression of steroidogenesis. Normally, when testosterone production is sufficiently inhibited, low-level testosterone acts on the negative feedback system of the HPT axis to stimulate LH output from the pituitary. LH promotes Leydig cells to synthesize and secrete more testosterone, which in the course of time, and acting via the same pathway, restores pituitary LH secretion to normal levels. In this manner, the negative feedback mechanism serves as a homeostatic control for the HPT axis [25]. However, our present study observed that plasma LH and testosterone levels in TCS-treated animals were simultaneously lower than that in the control, whereas no significant changes in GnRH levels were found; two vital receptors (LHR and $\mathrm{AR}$ ) in the testis were also depressed in TCS-exposed rats, data above indicating that the normal feedback mechanism of the HPT axis was disrupted after TCS treatments.

Testosterone is a C19 $\Delta^{4}-3-$ ketosteroid and irreversibly reduced to $5 \alpha$-dihydrotestosterone ( $5 \alpha$-DHT) in the male reproductive tract by two wellcharacterized $5 \alpha$-Reductases including $5 \alpha$-Reductase type 1 and type 2 that are encoded by SRD5A1 and SRD5A2, respectively. Intriguingly, our current study observed that protein expressions of SRD5A1 and SRD5A2 in rat testes were downregulated by TCS in a dosedependent manner. We analyze that the decline in $5 \alpha$-Reductases may be the compensatory and protective mechanism, trying to limit irreversible conversions of testosterone to $5 \alpha$-DHT to ameliorate the adverse effects of TCS on testicular steroidogenesis. However, the possible compensatory mechanism varies from different endocrine disruptors. Our previous study found that SRD5A2 activity and protein expression were induced by di(2-ethylhexyl) phthalate (DEHP) to accelerate the irreversible conversion of testosterone, contributing to the decrease in testosterone levels [16]. Nevertheless, the similar compensatory mechanism has appeared in perfluorooctane sulfonate (PFOS) and perfluorooctanoic acid (PFOA) exposures. PFOS and PFOA competitively depressed rat SRD5A1 activity, thereby controlling the biosynthesis of neurosteroids [26].

\section{Conclusion}

Our current study indicates that the miR-6321/Map3k1-regulated JNK/c-Jun/Nur77 cascade contributes to TCS-caused inhibition of testicular steroidogenesis (Fig. 7), and the disruption of HPT axis is also responsible for declines in testosterone caused by TCS. With respect to the downregulation in $5 \alpha$-Reductase expressions, it could be considered 


\section{Cellular Physiology Cell Physiol Biochem 2018;50:2029-2045 \begin{tabular}{l|l|l|l|l} 
DO 2018 The Author(s). Published by S. Karger AG, Basel \\
and Biochemistry
\end{tabular} \\ Ha et al.: The miR-6321/JNK/Nur77 Inhibits Steroidogenesis}

as a compensatory mechanism to alleviate the detrimental effects of TCS on testicular steroidogenesis. This study will contribute to a better understanding of the anti-androgenic properties of TCS, as well as its mechanisms of action.

\section{Acknowledgements}

This work was funded by the Chongqing Population and Family Planning Science and Technology Research Institute (2016cstc-jbky-01701). We thank Dr. De-Sheng Pei to polish the manuscript.

\section{Disclosure Statement}

The authors declare that they have no conflict of interests.

\section{References}

1 Toms LM, Allmyr M, Mueller JF, Adolfsson-Erici M, McLachlan M, Murby J, Harden FA: Triclosan in individual human milk samples from Australia. Chemosphere 2011;85:1682-1686.

2 Philippat C, Wolff MS, Calafat AM, Ye X, Bausell R, Meadows M, Stone J, Slama R, Engel SM: Prenatal exposure to environmental phenols: concentrations in amniotic fluid and variability in urinary concentrations during pregnancy. Environ Health Perspect 2013;121:1225-1231.

-3 Allmyr M, Harden F, Toms LM, Mueller JF, McLachlan MS, Adolfsson-Erici M, Sandborgh-Englund G: The influence of age and gender on triclosan concentrations in Australian human blood serum. Sci Total Environ 2008;393:162-167.

-4 Hwang J, Suh SS, Park M, Park SY, Lee S, Lee TK: Differential gene expression patterns during embryonic development of sea urchin exposed to triclosan. Environ Toxicol 2017;32:426-433.

-5 Zhang P, Yang M, Zeng L, Liu C: P38/TRHr-dependent regulation of TPO in thyroid cells contributes to the hypothyroidism of triclosan-treated rats. Cell Physiol Biochem 2018;45:1303-1315.

6 Jackson-Browne MS, Papandonatos GD, Chen A, Calafat AM, Yolton K, Lanphear BP, Braun JM: Identifying vulnerable periods of neurotoxicity to triclosan exposure in children. Environ Health Perspect 2018;126:057001.

7 Narrowe AB, Albuthi-Lantz M, Smith EP, Bower KJ, Roane TM, Vajda AM, Miller CS: Perturbation and restoration of the fathead minnow gut microbiome after low-level triclosan exposure. Microbiome 2015;3:6.

-8 Jurewicz J, Radwan M, Wielgomas B, Kałużny P, Klimowska A, Radwan P, Hanke W: Environmental levels of triclosan and male fertility. Environ Sci Pollut Res Int 2018;25:5484-5490.

-9 Wang F, Liu F, Chen W, Xu R, Wang W: Effects of triclosan (TCS) on hormonal balance and genes of hypothalamus-pituitary-gonad axis of juvenile male Yellow River carp (Cyprinus carpio). Chemosphere 2018;193:695-701.

10 Wang CF, Tian Y: Reproductive endocrine-disrupting effects of triclosan: population exposure, present evidence and potential mechanisms. Environ Pollut 2015;206:195-201.

-11 Kumar V, Chakraborty A, Kural MR, Roy P: Alteration of testicular steroidogenesis and histopathology of reproductive system in male rats treated with triclosan. Reprod Toxicol 2009;27:177-185.

$\checkmark 12$ Kumar V, Balomajumder C, Roy P: Disruption of LH-induced testosterone biosynthesis in testicular Leydig cells by triclosan: probable mechanism of action. Toxicology 2008;250:124-131.

13 Feng Y, Zhang P, Zhang Z, Shi J, Jiao Z, Shao B: Endocrine disrupting effects of triclosan on the placenta in pregnant rats. PLoS One 2016;11:e0154758.

14 Zheng D, Zhao Y, Shen Y, Chang X, Ju S, Guo L: Orexin A-mediated stimulation of 3 $\beta$-HSD expression and estosterone production through MAPK signaling pathways in primary rat Leydig cells. J Endocrinol Invest 2014;37:285-292. 


\section{Cellular Physiology Cell Physiol Biochem 2018;50:2029-2045

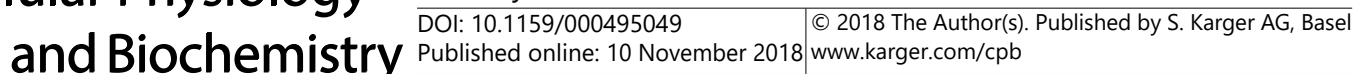

Ha et al.: The miR-6321/JNK/Nur77 Inhibits Steroidogenesis

15 Lai MS, Cheng YS, Chen PR, Tsai SJ, Huang BM: Fibroblast growth factor 9 activates Akt and MAPK pathways to stimulate steroidogenesis in mouse Leydig cells. PLoS One 2014;9:e90243.

16 Ha M, Guan X, Wei L, Li P, Yang M, Liu C: Di-(2-ethylhexyl) phthalate inhibits testosterone level through disturbed hypothalamic-pituitary-testis axis and ERK-mediated $5 \alpha$-Reductase 2. Sci Total Environ 2016;563-564:566-575.

17 Hu Z, Shen WJ, Kraemer FB, Azhar S: MicroRNAs 125a and 455 repress lipoprotein supported steroidogenesis by targeting scavenger receptor class B type I in steroidogenic cells. Mol Cell Biol 2012;32:5035-5045.

18 Geng XJ, Zhao DM, Mao GH, Tan L: MicroRNA-150 regulates steroidogenesis of mouse testicular Leydig cells by targeting STAR. Reproduction 2017;154:129-136.

19 Wang L, Xu C: Role of microRNAs in mammalian spermatogenesis and testicular germ cell tumors. Reproduction 2015;149:R127-R137.

20 Pratt SL, Calcatera SM: Expression of microRNA in male reproductive tissues and their role in male fertility. Reprod Fertil Dev 2016;29:24-31.

21 Ming S, Shui-Yun W, Wei Q Jian-Hui L, Ru-Tai H, Lei S, Mei J, Hui W, Ji-Zheng W: miR-139-5p inhibits isoproterenol-induced cardiac hypertrophy by targeting c-Jun. Biosci Rep 2018;38:BSR20171430.

22 Han YH, Cao X, Lin B, Lin F, Kolluri SK, Stebbins J, Reed JC, Dawson MI, Zhang XK: Regulation of Nur77 nuclear export by c-Jun N-terminal kinase and Akt. Oncogene 2006;25:2974-2986.

-23 Lee SY, Gong EY, Hong CY, Kim KH, Han JS, Ryu JC, Chae HZ, Yun CH, Lee K: ROS inhibit the expression of testicular steroidogenic enzyme genes via the suppression of Nur77 transactivation. Free Radic Biol Med 2009;47:1591-1600.

24 Sokanovic SJ, Janjic MM, Stojkov NJ, Baburski AZ, Bjelic MM, Andric SA, Kostic TS: Age related changes of cAMP and MAPK signaling in Leydig cells of Wistar rats. Exp Gerontol 2014;58:19-29.

-25 Akingbemi BT, Youker RT, Sottas CM, Ge R, Katz E, Klinefelter GR, Zirkin BR, Hardy MP: Modulation of rat Leydig cell steroidogenic function by di(2-ethylhexyl)phthalate. Biol Reprod 2001;65:1252-1259.

-26 Xu R, Shen Q, Li S, Li X, Li H, Lü Y, Lian QQ, Ge RS: Effects of perfluoroalkyl substances on neurosteroid synthetic enzymes in the rat. Chem Biol Interact 2017;272:182-187. 